Purdue University Purdue e-Pubs

$7-2002$

\title{
Investigation of the Vibrational Modes of Edge- Constrained Fibrous Samples Placed in a Standing Wave Tube
}

\author{
Bryan H. Song \\ NASA-Kennedy Space Center \\ J Stuart Bolton \\ Purdue University, bolton@purdue.edu
}

Follow this and additional works at: http://docs.lib.purdue.edu/herrick

Song, Bryan H. and Bolton, J Stuart, "Investigation of the Vibrational Modes of Edge-Constrained Fibrous Samples Placed in a Standing Wave Tube" (2002). Publications of the Ray W. Herrick Laboratories. Paper 78.

http://docs.lib.purdue.edu/herrick/78

This document has been made available through Purdue e-Pubs, a service of the Purdue University Libraries. Please contact epubs@purdue.edu for additional information. 
INVESTIGATION OF THE VIBRATIONAL

MODES OF EDGE-CONSTRAINED

FIBROUS SAMPLES PLACED IN A

\section{STANDING WAVE TUBE}

\section{Bryan H. Song}

NASA-Kennedy Space Center

J. Stuart Bolton

Ray W. Herrick Laboratories

Purdue University

\section{Purdue University}




\section{Background}

- Measurement of the surface normal velocity of vibrating panels or plates [Kruger and Mann (1999) and Muller and Moslehy (1996)]

- Estimation of the mechanical properties (Young's modulus and Poisson's ratio) of foam materials by the laser vibration measurement [Mariez et al. (1996) and Dubbelday (1992)]

- Electromagnetic approach for measuring the vibrational velocity of the frame of flexible porous materials (Khirnykh and Cummings, 1999) 


\section{Introduction}

- Investigation of edge constraint effect on samples placed in a modified standing wave tube (J. S. Bolton et al., SAE 1997; B. H. Song et al., JASA 1999).

- Internal constraints may be used to selectively enhance the transmission loss of lining materials at low frequencies $(B$. H. Song et al., JASA 2001).

- Implications for design of low frequency noise control barriers following from constraint of porous lining materials around their edges. 


\section{Glass Fiber Material inside of Sample Holder}

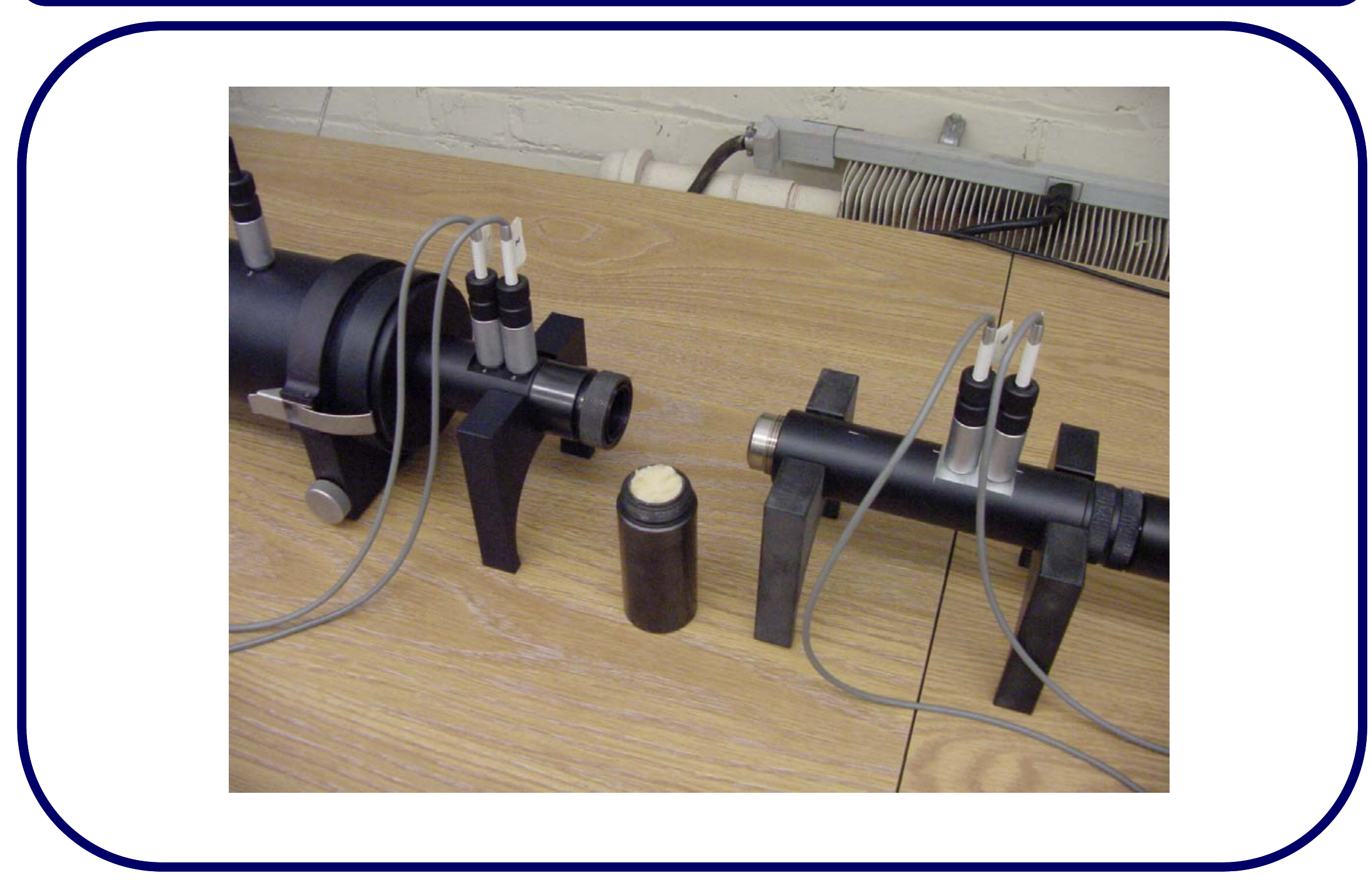




\section{Four Microphone Measurement}

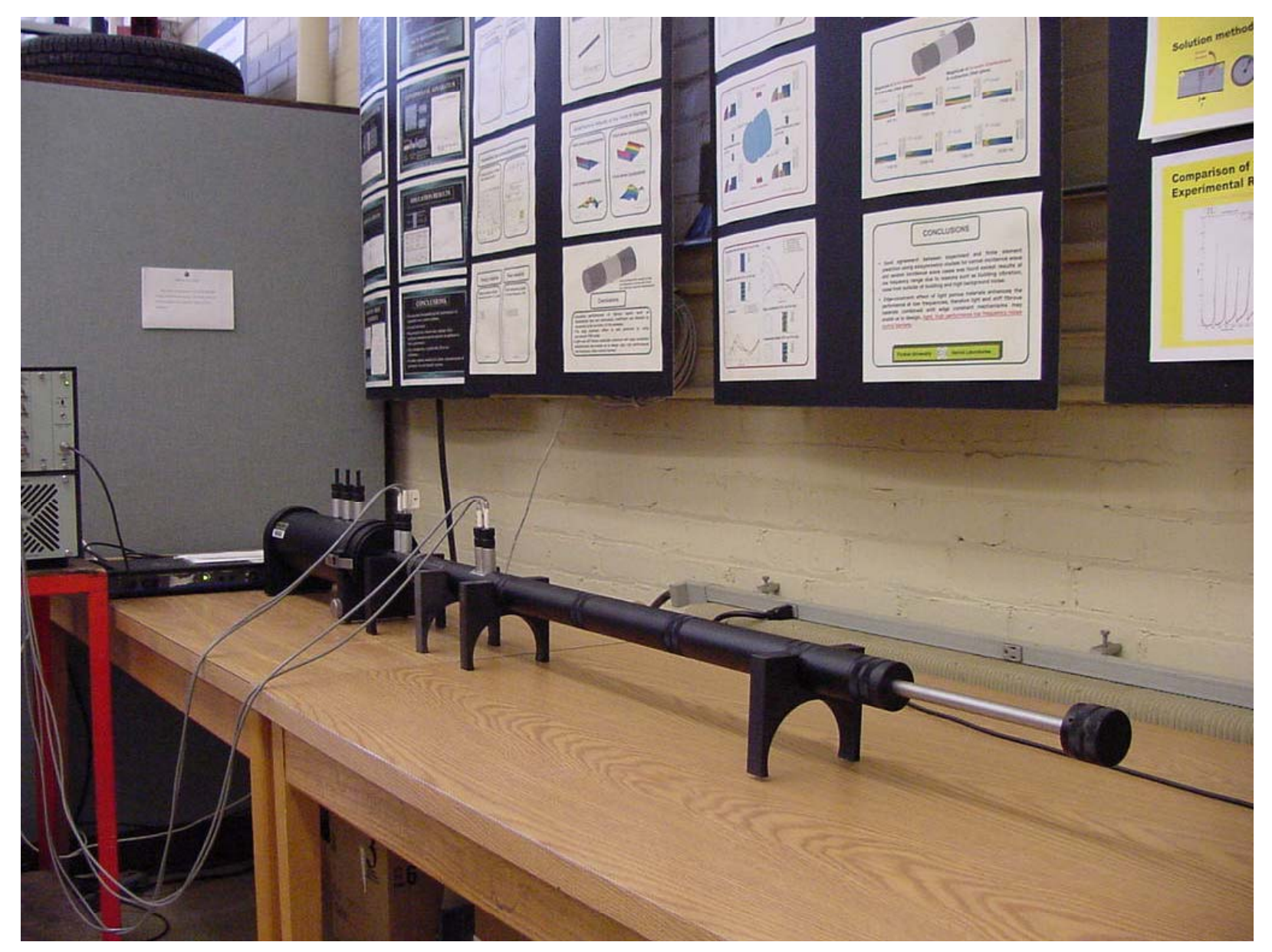




\section{Transfer Matrix Approach I}

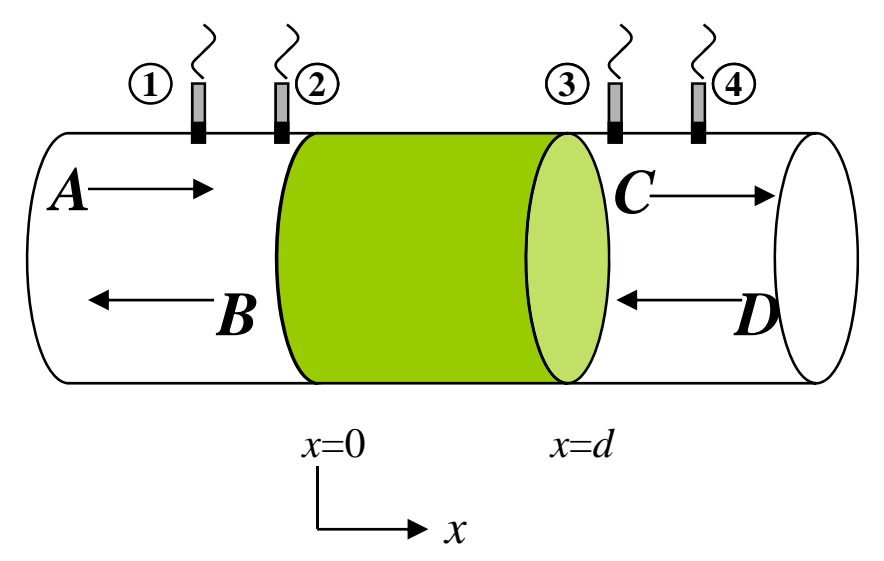

$\left[\begin{array}{l}P \\ V\end{array}\right]_{x=0}=\left[\begin{array}{ll}T_{11} & T_{12} \\ T_{21} & T_{22}\end{array}\right]\left[\begin{array}{l}P \\ V\end{array}\right]_{x=d}$

$T_{11}=T_{22}$

$\longrightarrow$ symmetry

$T_{11} T_{22}-T_{12} T_{21}=1$

$\square$ reciprocity

- Solve for transfer matrix elements 


\section{Transfer Matrix Approach II}

$$
\left[\begin{array}{c}
1+R_{a} \\
\frac{1-R_{a}}{\rho_{0} C_{0}}
\end{array}\right]=\left[\begin{array}{ll}
T_{11} & T_{12} \\
T_{21} & T_{22}
\end{array}\right]\left[\begin{array}{c}
T_{a} e^{-j k d} \\
\frac{T_{a} e^{-j k d}}{\rho_{0} c_{0}}
\end{array}\right]
$$

- Anechoic Reflection Coefficient

$$
R_{a}=\frac{T_{11}+\frac{T_{12}}{\rho_{0} c}-\rho_{0} c T_{21}-T_{22}}{T_{11}+\frac{T_{12}}{\rho_{0} c}+\rho_{0} c T_{21}+T_{22}} \Longrightarrow \begin{aligned}
& \alpha=1-\left|R_{a}\right|^{2} \\
& \frac{Z_{n}}{\rho_{0} c}=\frac{1+R_{a}}{1-R_{a}}
\end{aligned}
$$

- Anechoic Transmission Coefficient

$$
T_{a}=\frac{2 e^{j k d}}{T_{11}+\frac{T_{12}}{\rho_{0} c}+\rho_{0} c T_{21}+T_{22}}
$$$$
\Longrightarrow T L=10 \log \left(1 /\left|T_{a}\right|^{2}\right)
$$ 


\section{Experimental Setup for Low Frequency Tube}

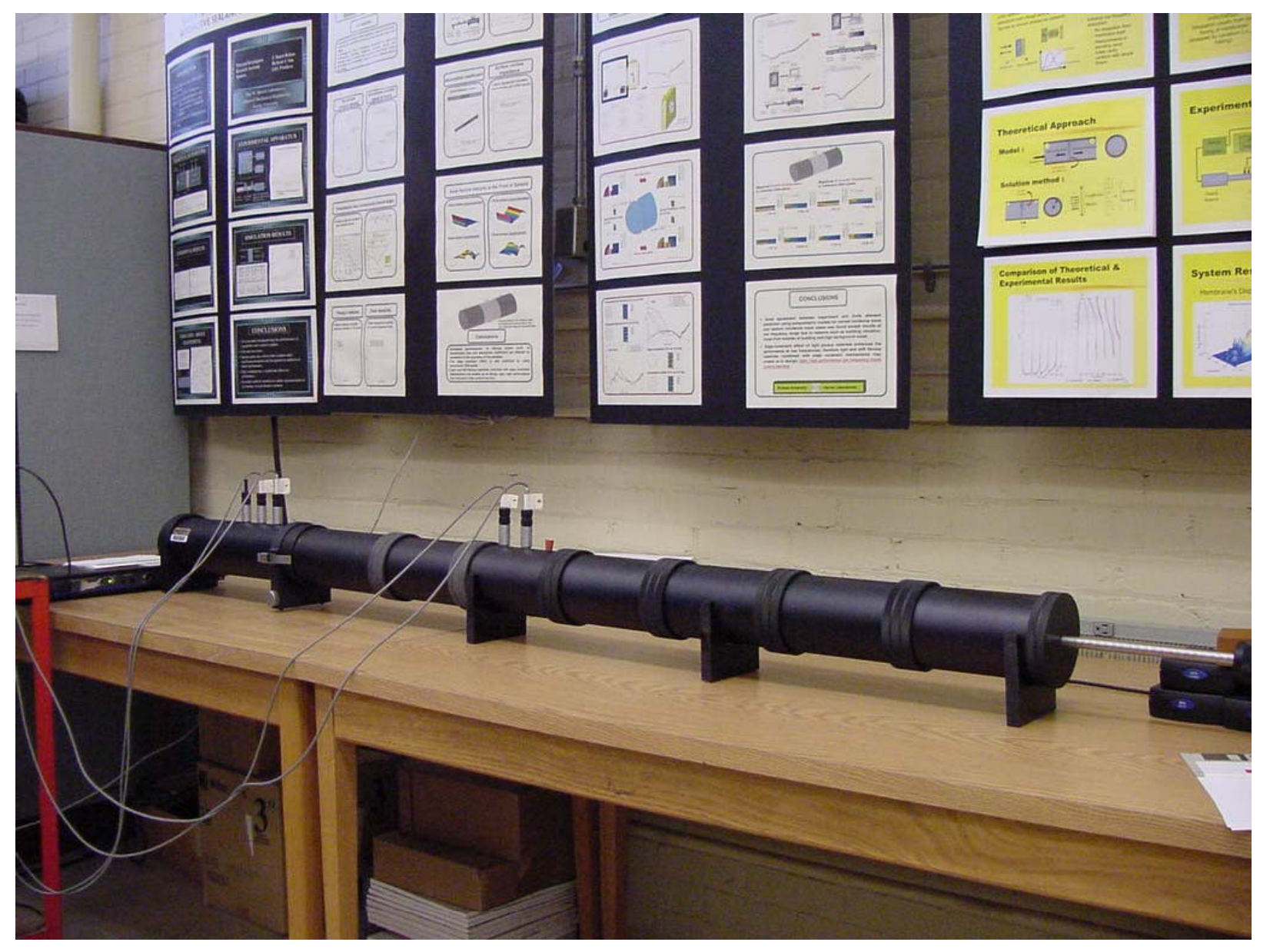




\section{Anechoic Transmission Loss (3" Sample A in a Large Tube)}

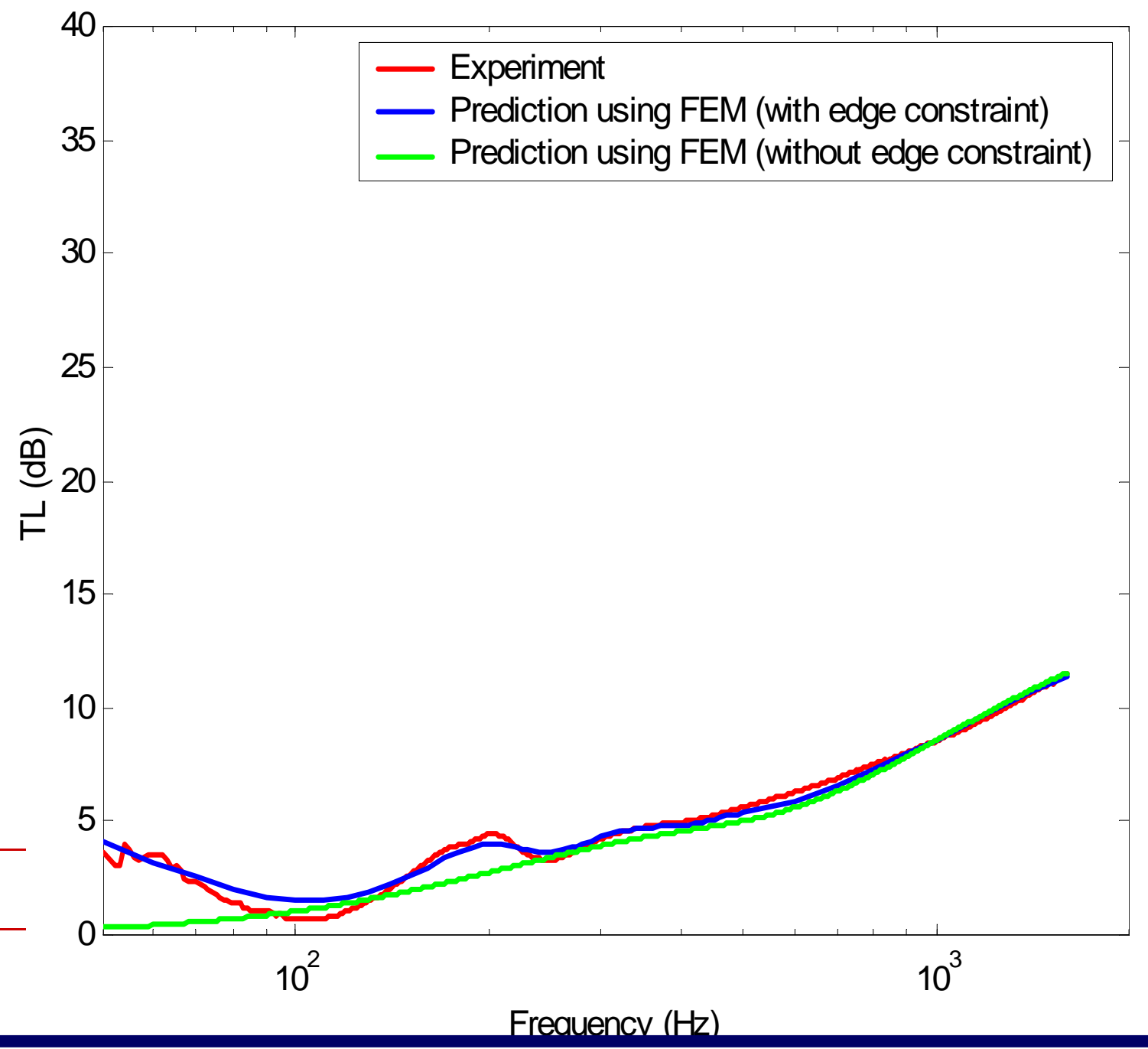




\section{Surface Normal Impedance \\ (3" Sample A in a Large Tube)}
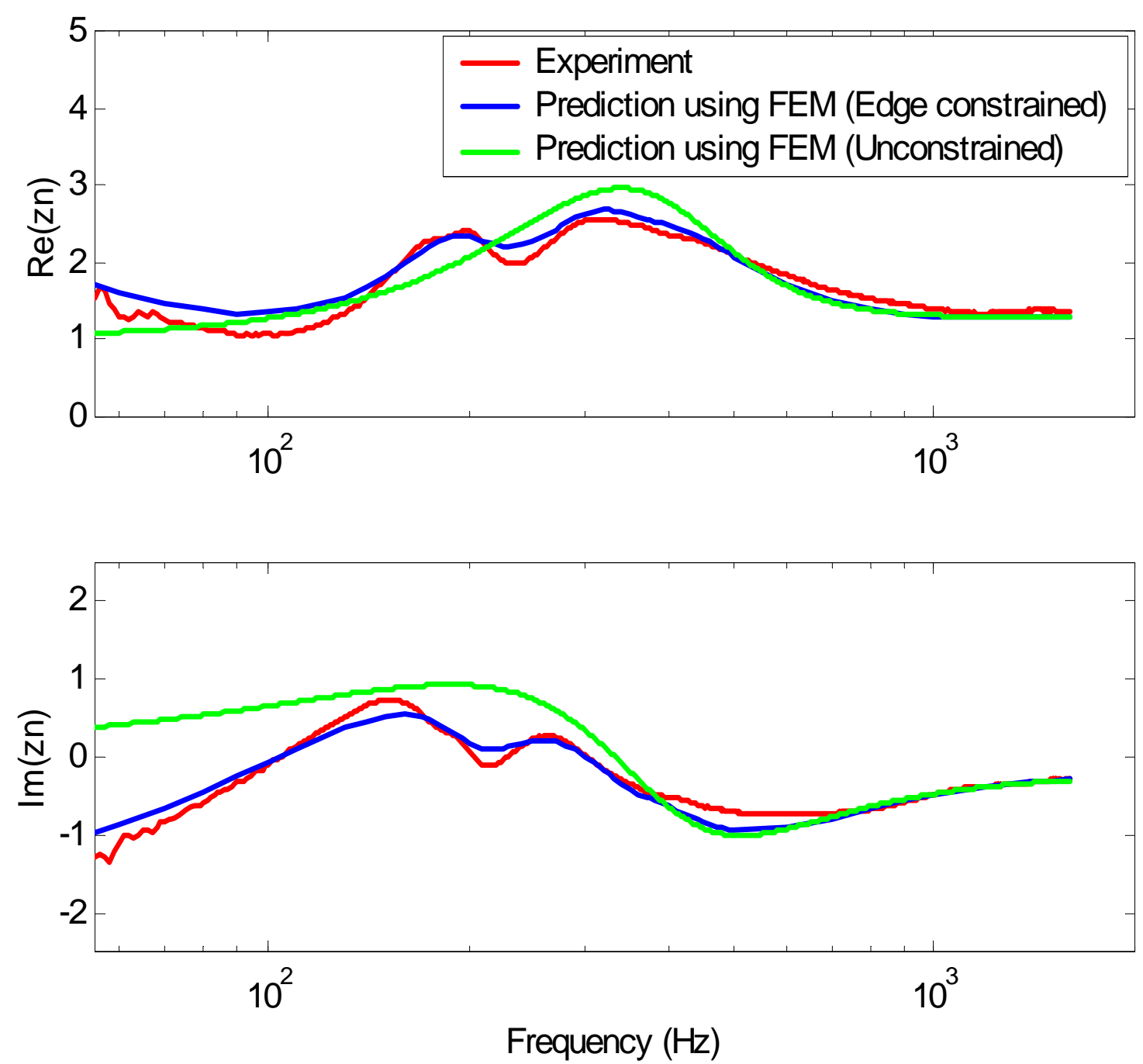


\section{Poroelastic Material Properties used in Calculations}

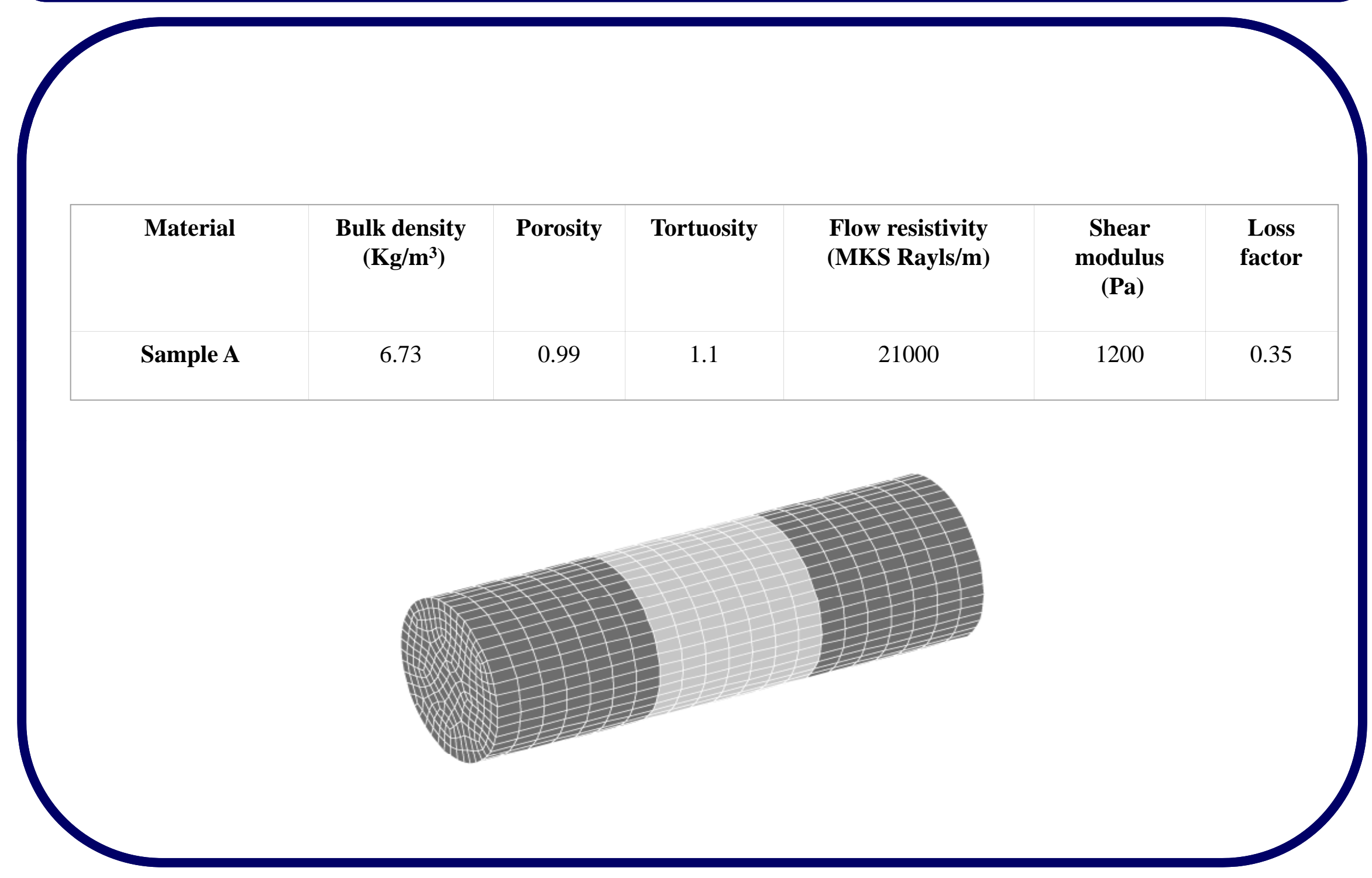




\section{Investigation of Vibrational Modes of Glass Fiber Materials (1" Sample A)}

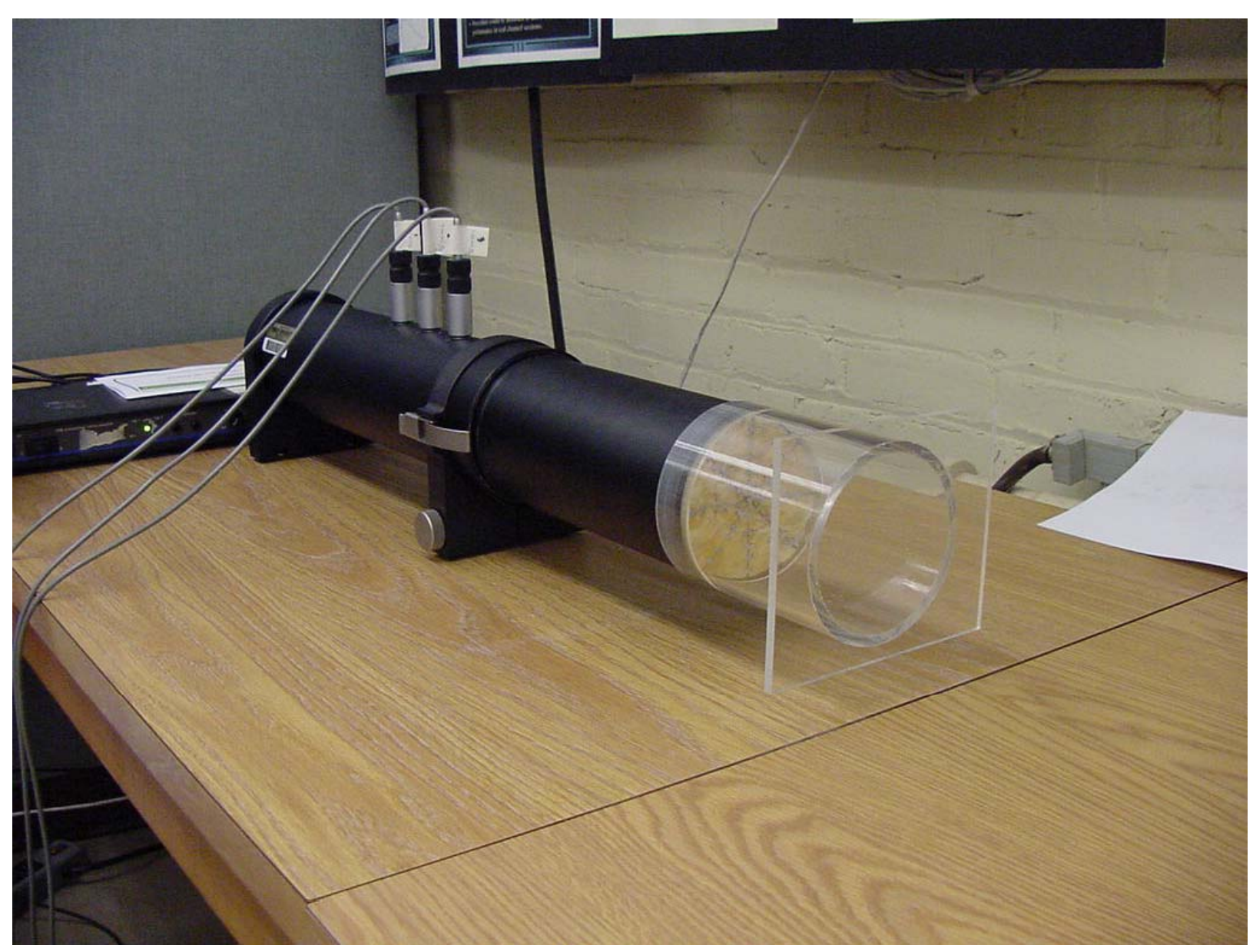




\section{Laser Measurement Setup (Large Tube, 1" Sample A)}

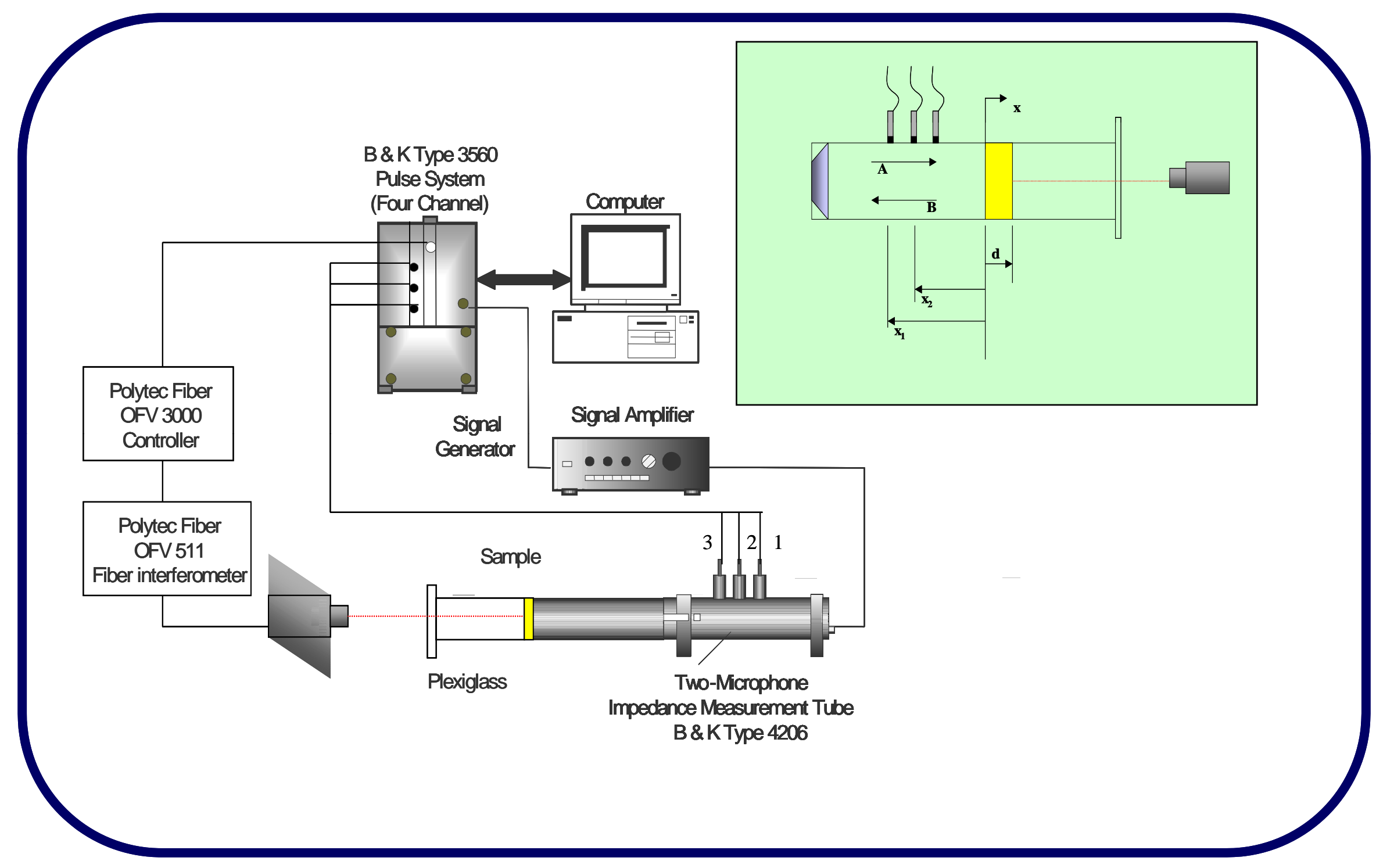




\section{Three-Dimensional Finite Element Model}

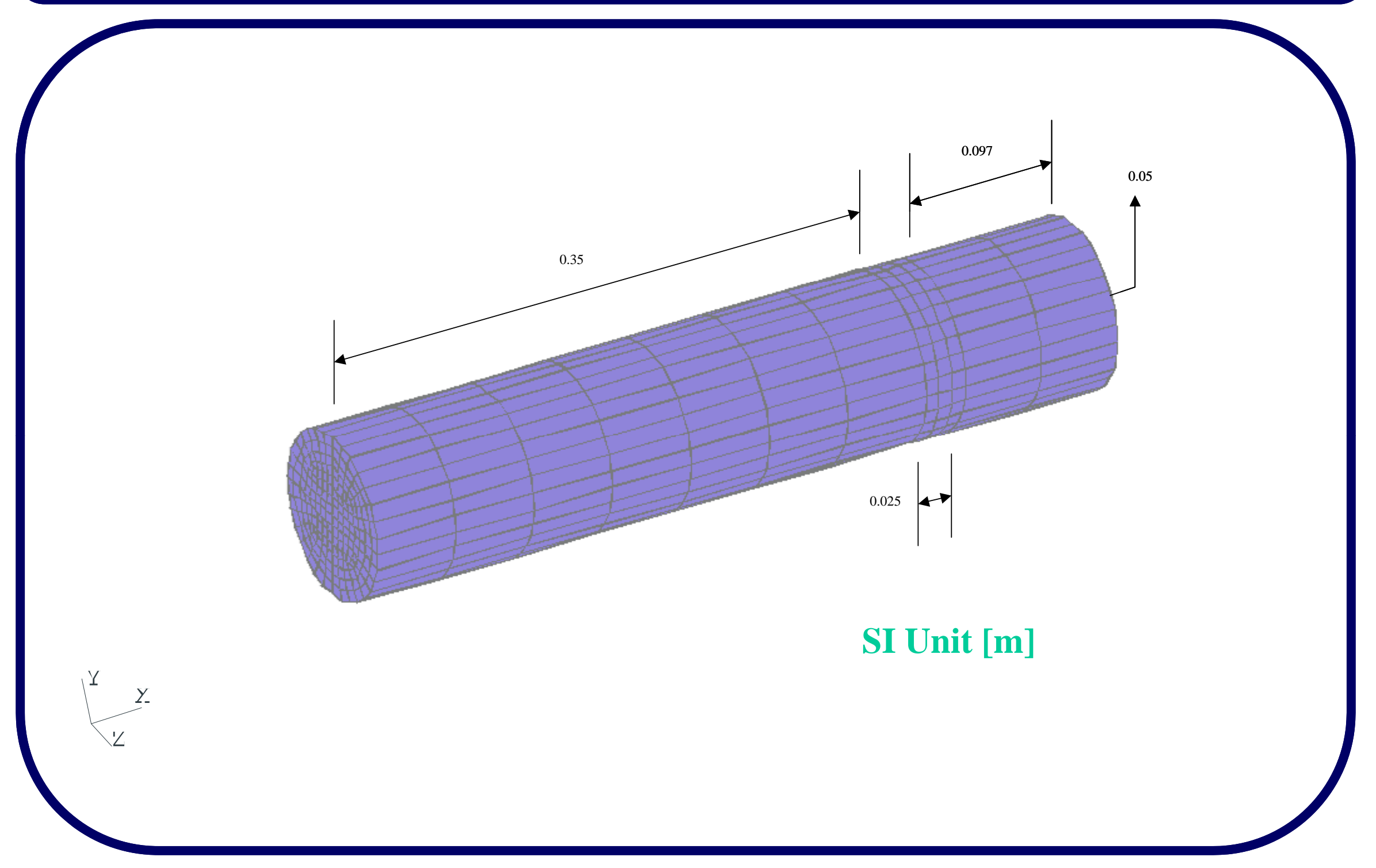




\section{Schematic of Edge- and Plane-Constrained Sample with Reflecting Tapes}

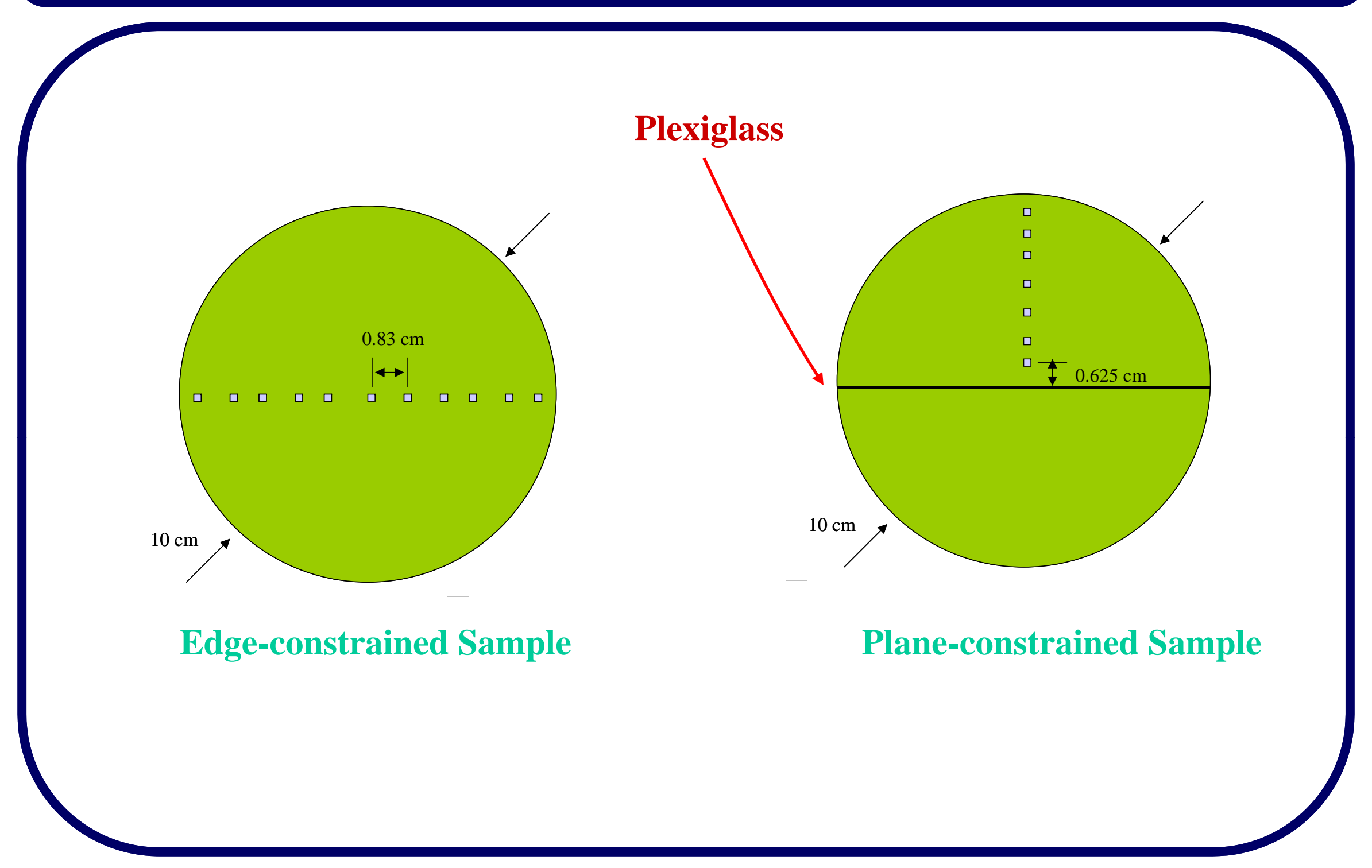


Glass Fiber Materials with Reflecting Tape

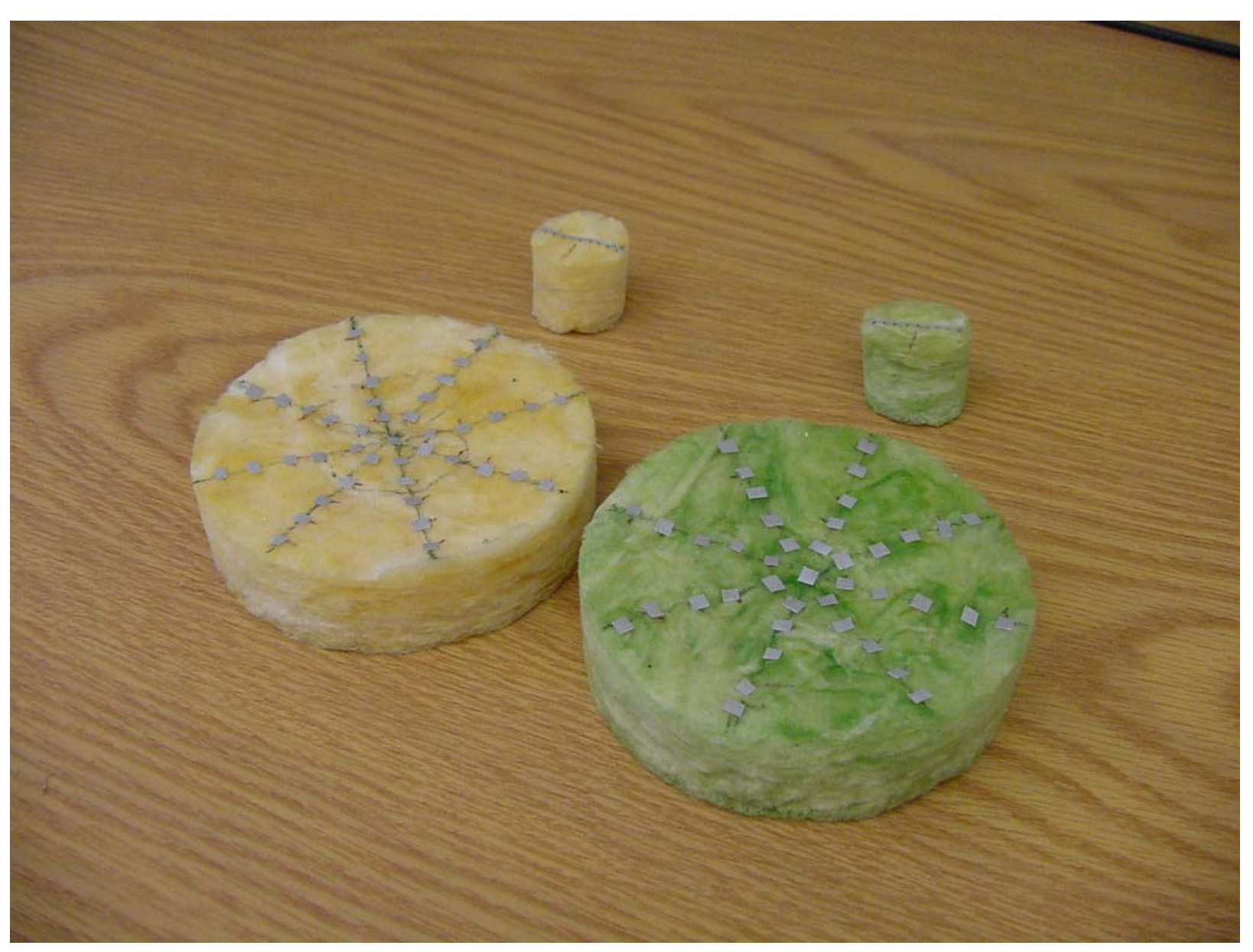




\section{Effect of Reflecting Tape on TL (1" Sample A)}

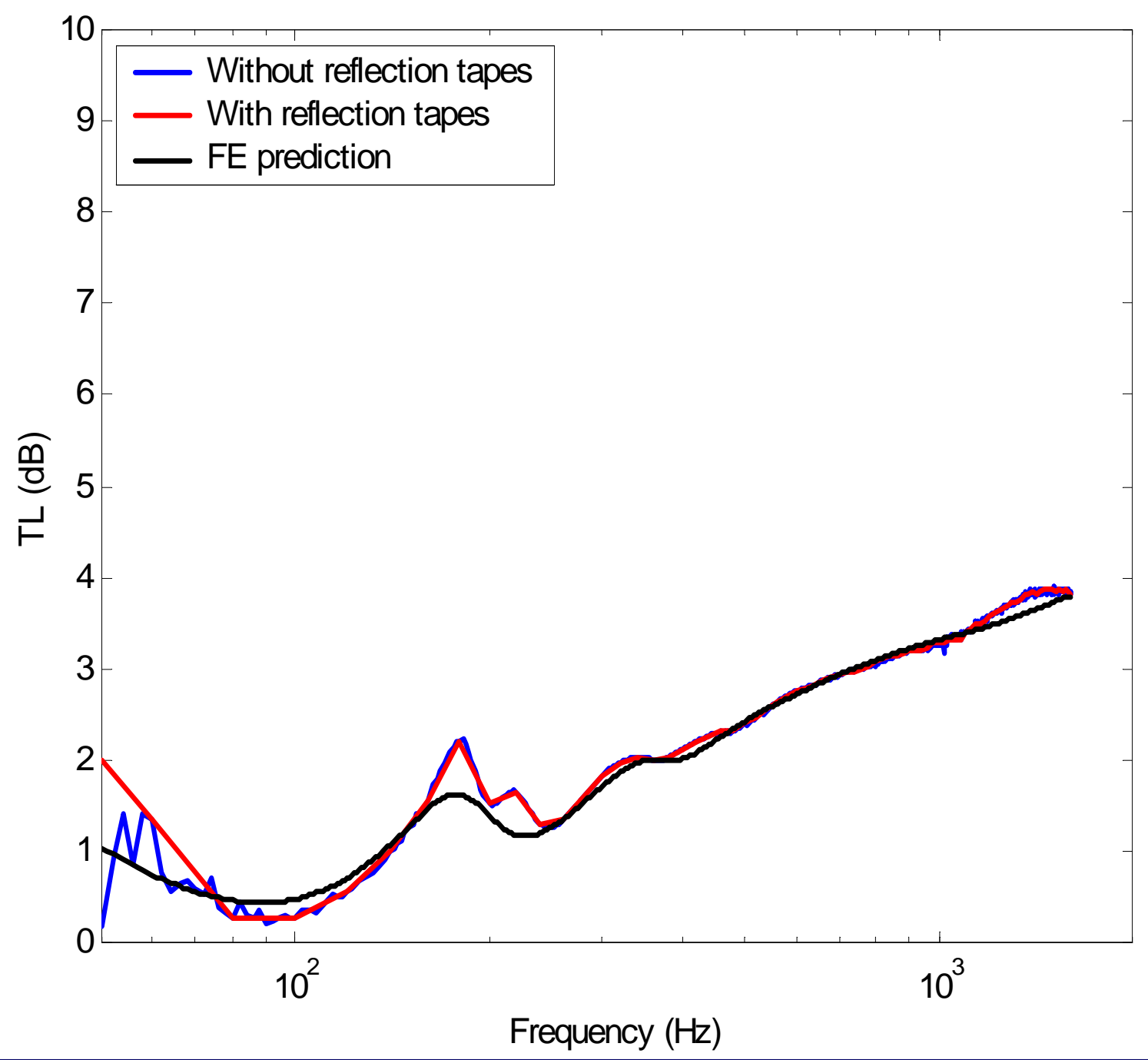




\section{Coherence Between Reference Microphone and the Laser Velocity Signal (1" Sample A)}

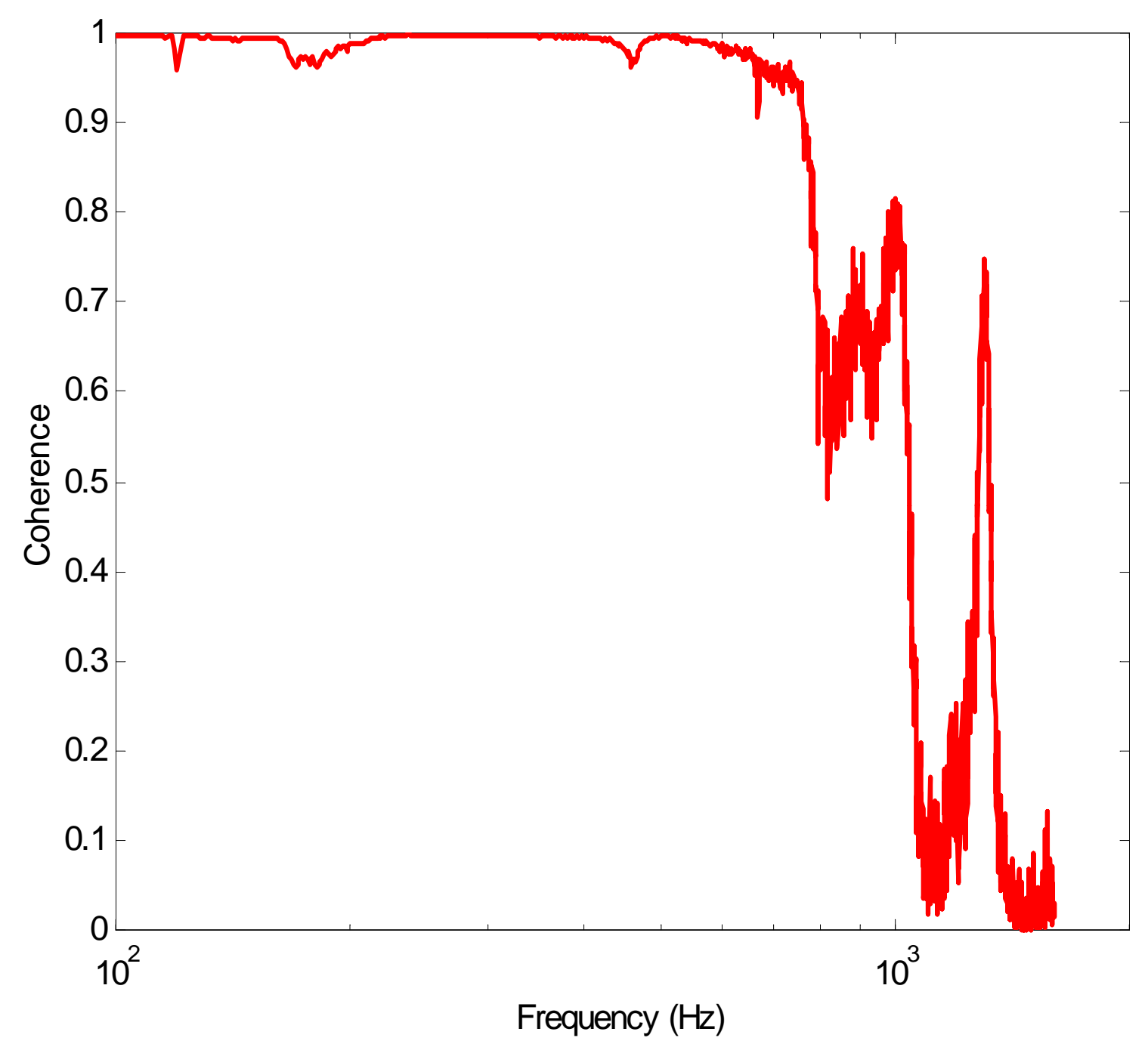




\section{FEM-Predicted Normalized Frame Velocity in Large Tube, Anechoic Termination Case (1")}

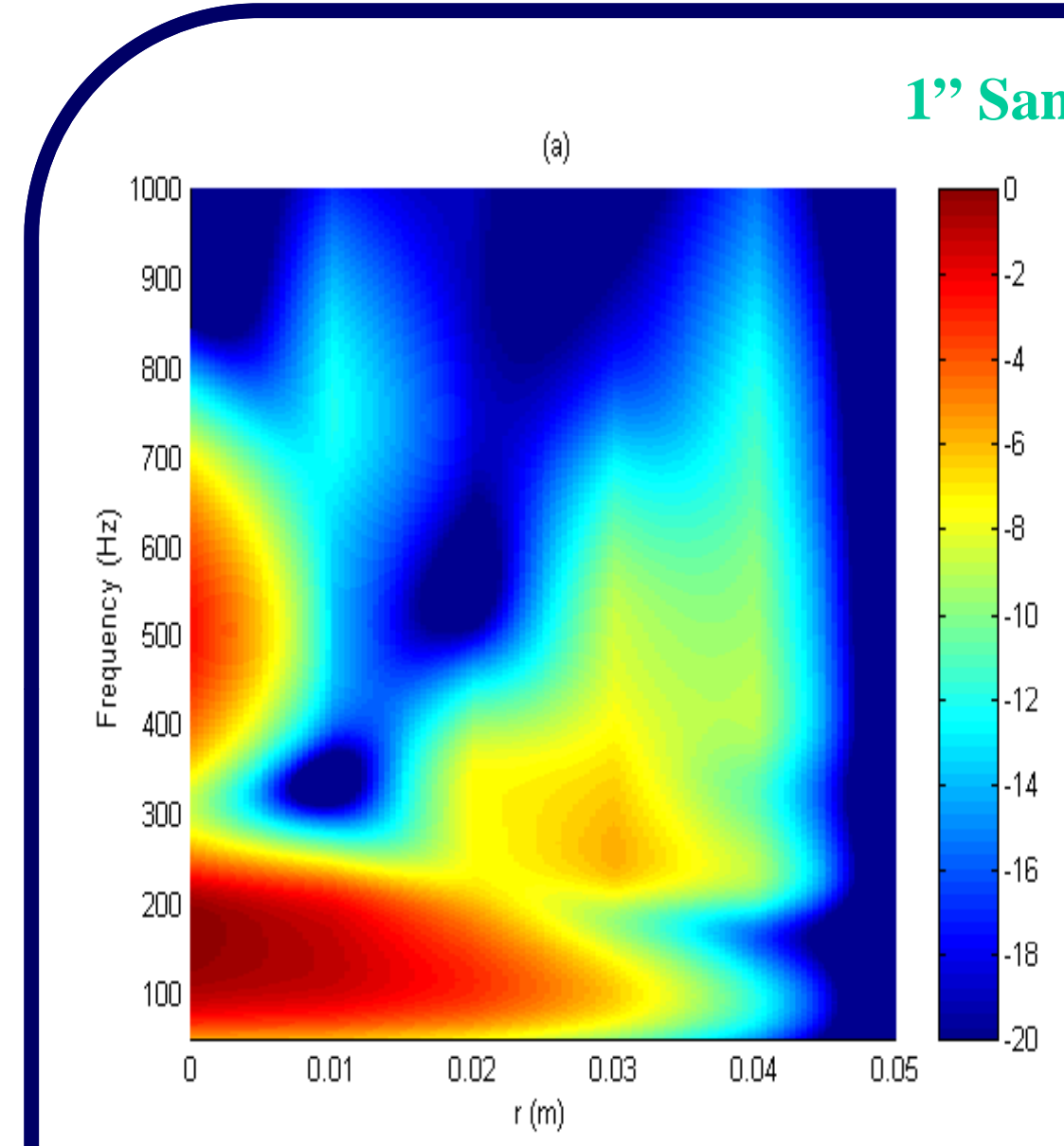

Edge-constrained Sample

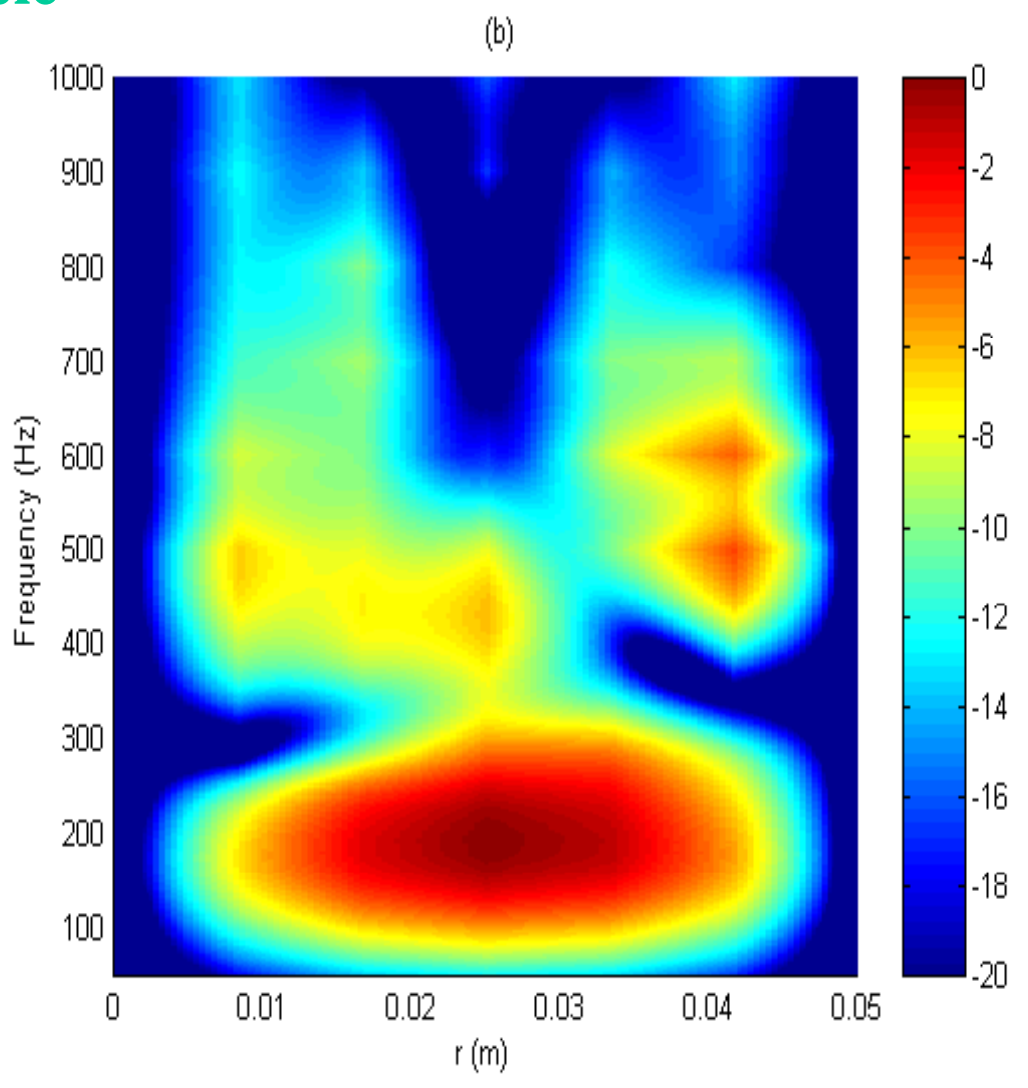

Plane-constrained Sample 


\section{The $1^{\text {st }}$ and $2^{\text {nd }}$ Mode Shapes of the Edge-constrained Sample (1")}

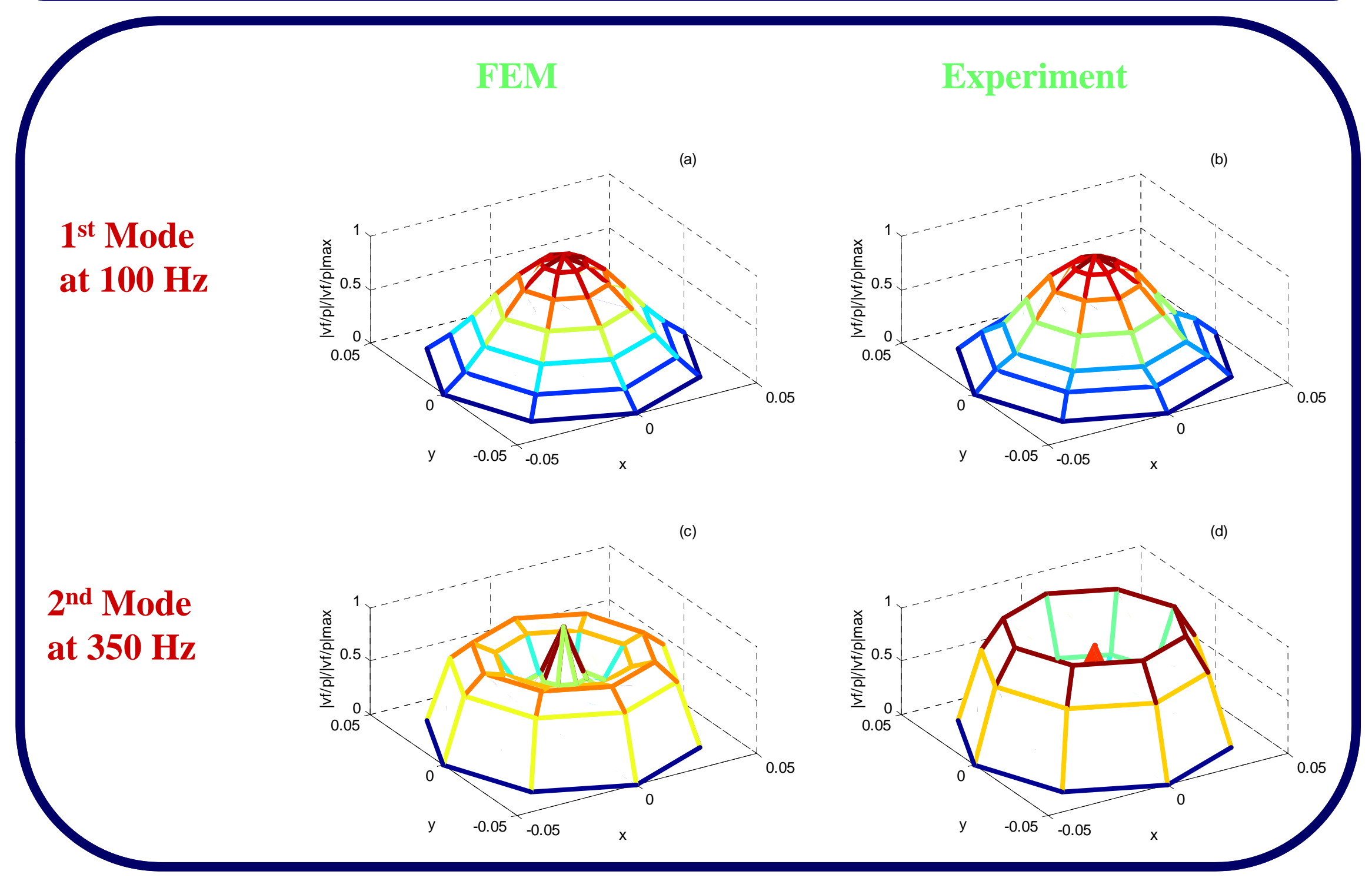




\section{The $1^{\text {st }}$ and $2^{\text {nd }}$ Mode Shapes of the Plane-constrained Sample (1")}

$1^{\text {st }}$ Mode at $200 \mathrm{~Hz}$

$2^{\text {nd }}$ Mode at $500 \mathrm{~Hz}$
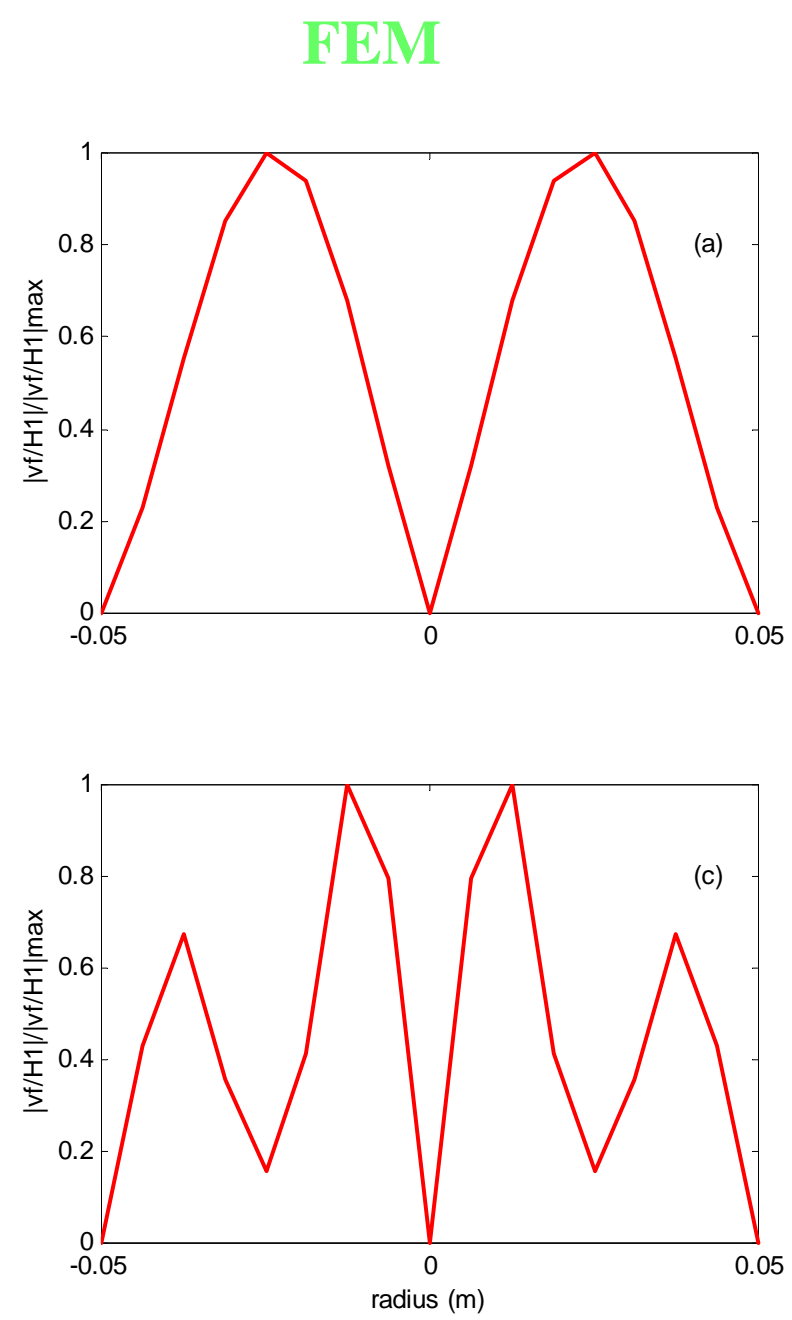

Experiment
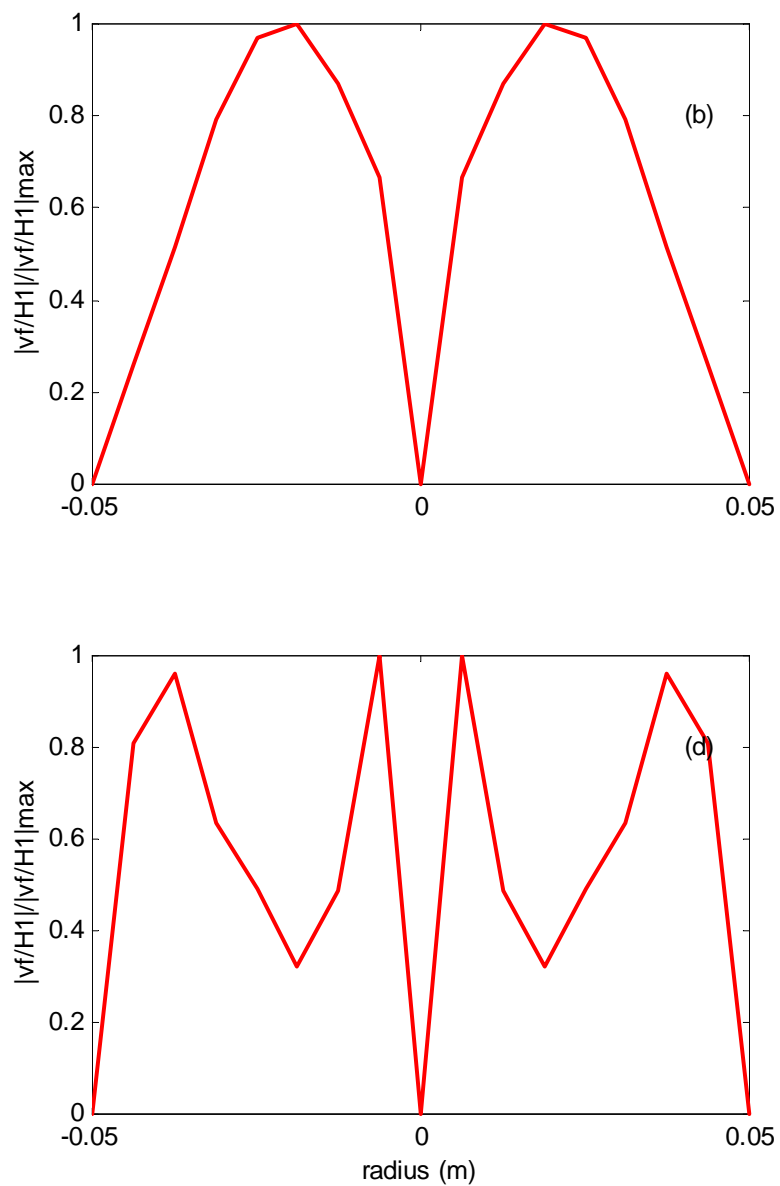


\section{Normalized Frame Velocity of Sample in Large Tube, Hard Termination Case (1")}

Edgeconstrained

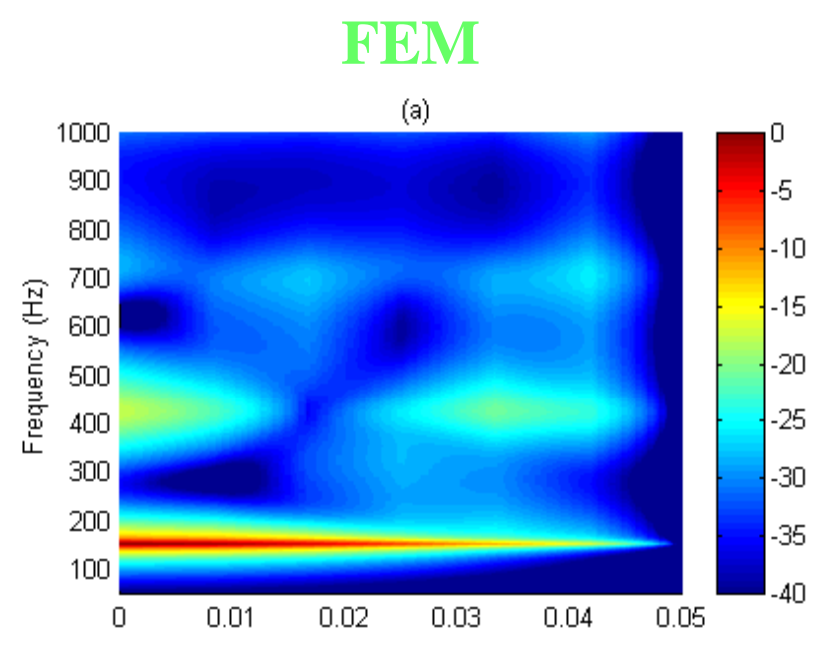

Plane-

constrained
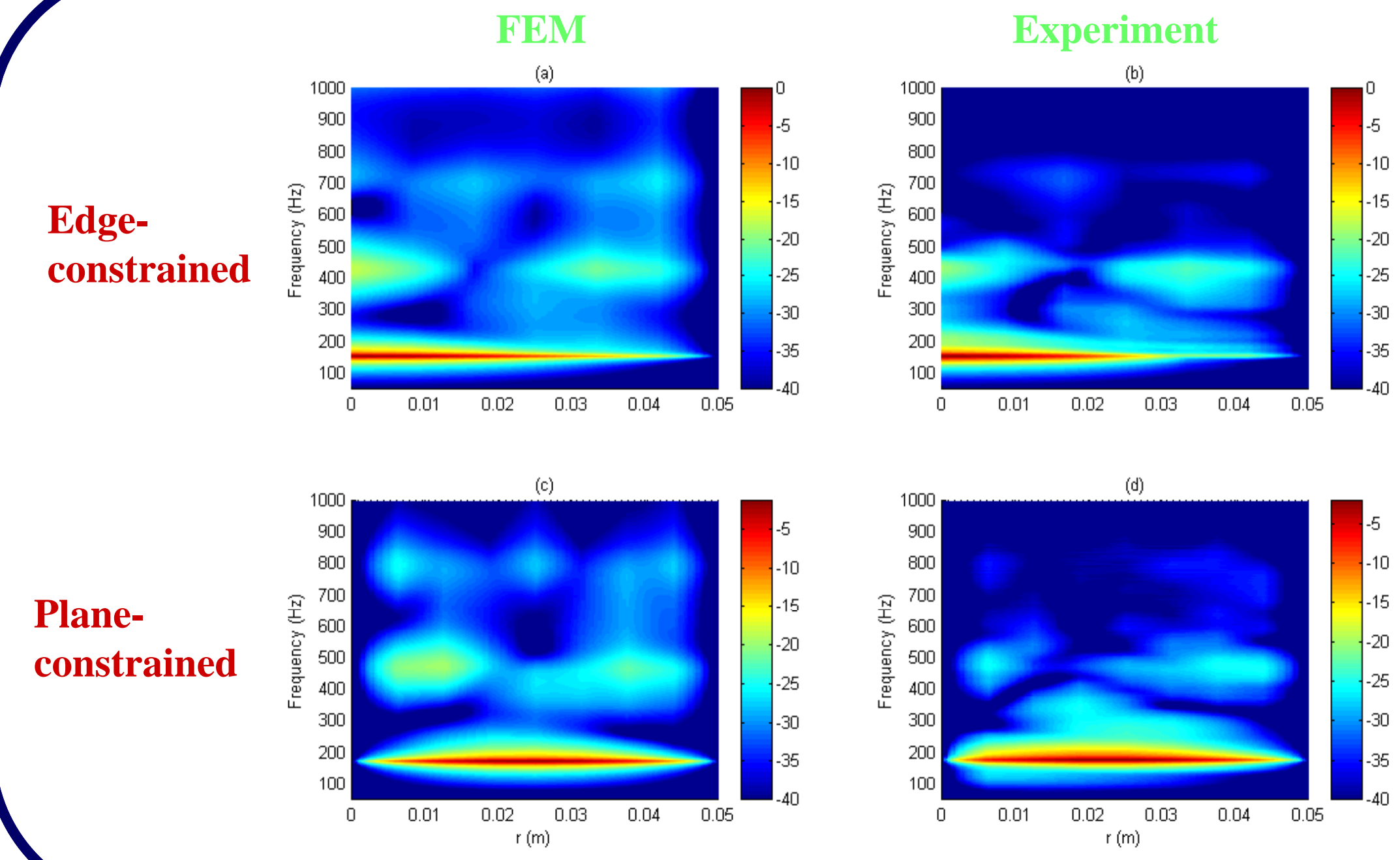


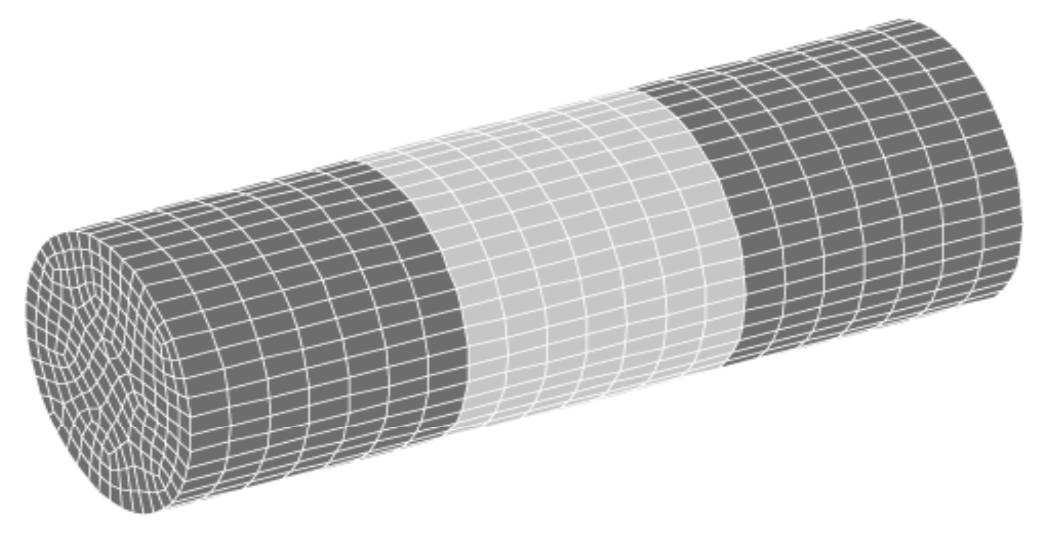

\section{Conclusions}

- Direct measurements show the vibrational velocity of the solid skeleton of fibrous sample placed in a standing wave tube.

- The vilbrational characteristics are well predicted by using poroelastic FE model (COMET/SAFE).

- Vibrational modes of edge- and plane-constrained fibrous layers have been verified using a laser vibrometer measurement. 